\title{
Direct discrete time design of robust state derivative feedback control laws
}

\section{Fernanda Quelho Rossi, Roberto Kawakami Harrop Galvão, Marcelo Carvalho Minhoto Teixeira \& Edvaldo Assunção}

To cite this article: Fernanda Quelho Rossi, Roberto Kawakami Harrop Galvão, Marcelo Carvalho Minhoto Teixeira \& Edvaldo Assunção (2018) Direct discrete time design of robust state derivative feedback control laws, International Journal of Control, 91:1, 70-84, DOI: 10.1080/00207179.2016.1266521

To link to this article: https://doi.org/10.1080/00207179.2016.1266521

Accepted author version posted online: 29

Nov 2016.

Published online: 20 Dec 2016.

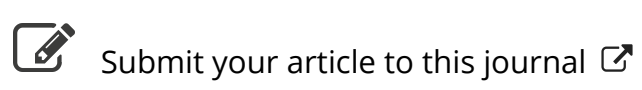

Џll Article views: 228

View Crossmark data ¿

Citing articles: 3 View citing articles 5 


\title{
Direct discrete time design of robust state derivative feedback control laws
}

\author{
Fernanda Quelho Rossi $\odot^{\mathrm{a}}$, Roberto Kawakami Harrop Galvão ${ }^{\mathrm{a}}$, Marcelo Carvalho Minhoto Teixeirab and \\ Edvaldo Assunção

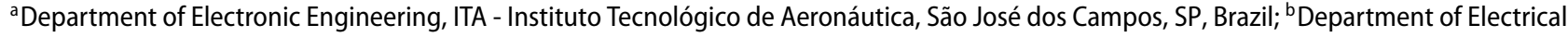 \\ Engineering, UNESP - Univ Estadual Paulista, Ilha Solteira, SP, Brazil
}

\begin{abstract}
This paper is concerned with the problem of designing robust state derivative feedback control laws in discrete time. The main contribution consists of a method for recasting a continuous time state space model in the form of a discrete time model formulated in terms of the state derivative. Uncertain input delays and parametric uncertainties in polytopic form can be propagated from the original state space representation to the resulting state derivative model. Therefore, robust control techniques originally developed for discrete time state space models can be directly employed to design the state derivative feedback law. Three computational examples are presented for illustration. The first example highlights the importance of accounting for the effect of sampling in the design procedure. More specifically, a linear quadratic regulation problem involving the state derivative is addressed. The second example involves the design of a robust predictive controller in the presence of input constraints and uncertain time delay. Finally, the third example is concerned with robust pole placement in the presence of parametric uncertainty.
\end{abstract}

\section{ARTICLE HISTORY}

Received 18 February 2016

Accepted 24 November 2016

\section{KEYWORDS}

State derivative feedback; direct discrete time design; robust control; robust predictive control; robust pole placement

\section{Introduction}

State derivative feedback control has received significant attention in the recent past, mainly in view of engineering applications where the state derivatives can be more easily obtained as compared to the actual state variables. Examples include active suspension devices (Reithmeier \& Leitmann, 2003; Silva, Assunção, Teixeira, \& Cardim, 2013), driving assistance controllers (Fallah, Khajepour, Fidan, Chen, \& Litkouhi, 2013) and vibration suppression systems (Abdelaziz \& Valášek, 2004), including applications in landing gear components (Kwak, Washington, \& Yedavalli, 2002), bridge cables (Duan, Ni, \& Ko, 2005), and earthquake hazard mitigation (Yang \& Li, 1991). In these cases, the state variables typically correspond to displacements and velocities. However, in the absence of an absolute position reference, the acceleration values are easier to obtain (Yang and $\mathrm{Li}, 1991$ ), since they can be measured by inertial accelerometers. The velocities and displacements can be estimated from the measured accelerations by single and double integration, respectively. However, the accuracy of the estimated displacements tends to be worse compared to the estimated velocities (Abdelaziz \& Valášek, 2004), due to the propagation of errors associated to bias in the acceleration measurements (Abdelaziz, 2013) and uncertainty in the initial conditions for the integrators (Reithmeier \&
Leitmann, 2003). Therefore, instead of using a state feedback control law expressed in terms of displacements and velocities, it may be more convenient to use state derivative feedback, which employs velocities and accelerations and does not require the estimation of the displacements.

Several contributions concerning state derivative feedback have been reported in the control literature. A pole placement method was proposed in Abdelaziz and Valášek (2004), with extensions for multivariable systems presented in subsequent works (Abdelaziz, 2009; Duan \& Yu, 2008; Faria, Assunção, Teixeira, Cardim, \& Silva, 2009). A linear quadratic regulator (LQR) framework was employed in Duan et al. (2005), Tseng and Hsieh (2013). Robustness issues have also been the subject of much research effort. Abdelaziz (2012) proposed an eigenstructure assignment method in which the available degrees of freedom were used to improve the robustness of the closed-loop system. A design method for uncertain systems employing linear matrix inequalities (LMIs) was presented in Assunção, Teixeira, Faria, Silva, \& Cardim (2007), with specifications on decay rate and bounds on the output peak. Subsequent extensions of this technique were concerned with D-stability specifications (Faria et al., 2009) and generalisations to descriptor systems (Faria, Assunção, Teixeira, \& Cardim, 2010). Less conservative LMI conditions were developed in Silva, Assunção, Teixeira, Faria, and Buzachero (2011), 
Silva, Assunção, Teixeira, and Buzachero (2012) by using parameter-dependent Lyapunov functions. LMI formulations for time delay systems have also been reported (Amri, Soudani, \& Benrejeb, 2011; Jing, Shen, Dimirovski, \& Jiang, 2009).

It is worth noting that most research efforts have been devoted to the design of continuous time controllers. To the authors' knowledge, the discrete time design of state derivative feedback controllers was only considered in Cardim, Teixeira, Faria, and Assunção (2009) and Rossi, Teixeira, Galvão, and Assunção (2013). The approach adopted in these two works consisted of first designing a digital state feedback controller by conventional means and then obtaining an equivalent digital state derivative feedback control law. However, such an equivalence was only valid for the nominal case, i.e. by considering a perfect match between the design model and the actual plant dynamics. In fact, a subsequent investigation (Rossi, Galvão, Teixeira, \& Assunção, 2015) revealed that the resulting state derivative feedback controller may actually have worse robustness properties compared to the original state feedback controller. As an example, the state derivative feedback controller in Rossi et al. (2015) was found to be less robust with respect to the introduction of time delay at the plant input. Within this scope, the lack of methods for direct design of robust discrete time state derivative feedback controllers is still an important gap in the literature.

In this context, the main contribution of the present paper consists of a method for recasting a continuous time state space model in the form of a discrete time model formulated in terms of the state derivative. The resulting model can be employed in a straightforward manner for the design of state derivative feedback controllers in discrete time, by using standard state space design techniques. Moreover, uncertain input delays and parametric uncertainties in polytopic form can be propagated from the original state space representation to the state derivative model, which allows the use of tools for robust control design.

For illustration, the proposed model formulation is employed in three computational examples involving well-known control design methods. The first example highlights the importance of accounting for sampling effects in the state derivative control law, by comparing LQRs designed with the continuous time model and the proposed discrete time model. A vibration suppression system is employed for illustration. In the second example, the same system is used to illustrate the design of robust model predictive control (RMPC) laws in the presence of input constraints and uncertain time delay. This second example is of particular interest within the context of robust state derivative feedback. In fact, to the best of the authors' knowledge, previous continuous time formulations were not capable of explicitly enforcing constraints on the control amplitude. Finally, the third example is concerned with robust pole placement for an active car seat suspension system in the presence of parametric uncertainty.

The remainder of this paper is organised as follows. Preliminary assumptions on the plant model are presented in Section 2, together with some details regarding the adopted discrete time notation. Section 3 states the main result involving the proposed discrete time state derivative model. The three illustrative design examples are presented in Section 4. Finally, concluding remarks are given in Section 5.

\section{Preliminaries and notation}

Consider a system described by a continuous time model of the form

$$
\dot{x}(t)=\Phi_{c} x(t)+\Gamma_{c} u(t)
$$

where $x(t) \in \mathbb{R}^{n}$ is the state vector, $u(t) \in \mathbb{R}^{m}$ is the control input and $\Phi_{c} \in \mathbb{R}^{n \times n}, \Gamma_{c} \in \mathbb{R}^{n \times m}$ are constant matrices, with $\Phi_{c}$ non-singular.

Remark 2.1: The invertibility of $\Phi_{c}$ is a standard assumption in the state derivative feedback literature, as can be seen for instance in Abdelaziz and Valášek (2004), Assunção et al. (2007), Duan et al. (2005), and Moreira et al. (2010).

Assume that the system is to be controlled by using sampled measurements of the state derivative $\dot{x}(k T), k \in$ $\mathbb{Z}$, where $T$ is the sampling period. Moreover, consider that a zero order hold is employed to keep the control $u(t)$ constant between sampling times, i.e.

$$
u(t)=u(k T)^{+}, \quad(k T)^{+} \leq t \leq(k+1) T
$$

The superscript + in (2) is employed to indicate that the control is updated immediately after the state derivative is measured at each sampling time, as in Rossi et al. (2013). The use of this notation is illustrated in Figure 1.

Therefore, the state derivative of the system (1) at time $t=k T$ is given by

$$
\dot{x}(k T)=\Phi_{c} x(k T)+\Gamma_{c} u((k-1) T)^{+}
$$

In what follows, definitions will be denoted by the symbol $\triangleq$. The notation $\operatorname{Co}\{\bullet\}$ will represent the convex hull of a set of vertices described in $\{\bullet\}$. A diagonal matrix will be denoted by $\operatorname{diag}\{\bullet\}$, with the diagonal elements indicated in $\{\bullet\}$. The squared norm of a vector $x$ weighted by 


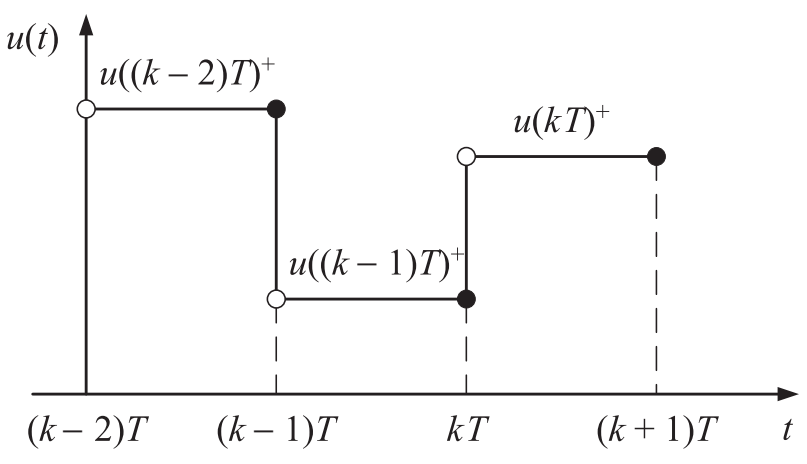

Figure 1. Update of the control input $u(t)$ at the sampling times.

a positive definite matrix $S$ of compatible dimensions will be denoted by $\|x\|_{S}^{2}=x^{T} S x$.

\section{Main result}

Since the control is kept constant between sampling times, as in Equation (2), the model (1) can be discretised as (Franklin, Powell, \& Workman, 1998)

$$
x((k+1) T)=\Phi x(k T)+\Gamma u(k T)^{+}
$$

with

$$
\begin{aligned}
& \Phi=e^{\Phi_{c} T}=\sum_{j=0}^{\infty}\left(\Phi_{c}\right)^{j} \frac{T^{j}}{j !} \\
& \Gamma=\int_{0}^{T} e^{\Phi_{c} \tau} \Gamma_{c} \mathrm{~d} \tau=\sum_{j=0}^{\infty}\left(\Phi_{c}\right)^{j} \frac{T^{(j+1)}}{(j+1) !} \Gamma_{c}
\end{aligned}
$$

The lemma below states a well-known result concerning the commutativity of matrices $\Phi$ and $\Phi_{c}$.

Lemma 3.1: Let $\Phi$ be given by (5). The product of matrices $\Phi$ and $\Phi_{c}$ is commutative, i.e.

$$
\Phi \Phi_{c}=\Phi_{c} \Phi
$$

Proof: The proof can be easily derived by using the power series expansion of $e^{\Phi_{c} T}$ in (5).

The following theorem shows that the model (4) can be reformulated in terms of the derivative of the state $\dot{x}(k T)$ and the control input, in a suitable form for use in discrete time control design.

Theorem 3.1: Let $\dot{x}(k T)$ denote the derivative of the state at sampling time $t=k T$, immediately before the control update. The discrete time model (4) can then be recast into the following form:

$$
\xi((k+1) T)=A \xi(k T)+B u(k T)^{+}
$$

with $\xi(k T) \in \mathbb{R}^{n+m}, \quad A \in \mathbb{R}^{(n+m) \times(n+m)} \quad$ and $\quad B \in$ $\mathbb{R}^{(n+m) \times m}$ defined as

$$
\begin{aligned}
\xi(k T) & \triangleq\left[\begin{array}{c}
\dot{x}(k T) \\
u((k-1) T)^{+}
\end{array}\right] \\
A & =\left[\begin{array}{cc}
\Phi & -\Phi \Gamma_{c} \\
0 & 0
\end{array}\right], \quad B=\left[\begin{array}{c}
\Phi \Gamma_{c} \\
I
\end{array}\right]
\end{aligned}
$$

where 0 and I denote a matrix of zeros and an identity matrix of appropriate dimensions, respectively.

Proof: In view of (1), the state derivative at time $t=k T$, immediately before the control update, is given by Equation (3), which is reproduced here for clarity:

$$
\dot{x}(k T)=\Phi_{c} x(k T)+\Gamma_{c} u((k-1) T)^{+}
$$

By replacing $k$ with $k+1$ in (11), one obtains

$$
\dot{x}((k+1) T)=\Phi_{c} x((k+1) T)+\Gamma_{c} u(k T)^{+}
$$

From (4) and (12), it follows that

$$
\dot{x}((k+1) T)=\Phi_{c} \Phi x(k T)+\left(\Phi_{c} \Gamma+\Gamma_{c}\right) u(k T)^{+}
$$

Assuming that $\Phi_{c}$ is non-singular (as stated in Section 2), Equation (11) can be rewritten as

$$
x(k T)=\Phi_{c}^{-1} \dot{x}(k T)-\Phi_{c}^{-1} \Gamma_{c} u((k-1) T)^{+}
$$

From (13) and (14), one obtains

$$
\begin{aligned}
\dot{x}((k+1) T)= & \Phi_{c} \Phi \Phi_{c}^{-1} \dot{x}(k T)-\Phi_{c} \Phi \Phi_{c}^{-1} \Gamma_{c} u((k-1) T)^{+} \\
& +\left(\Phi_{c} \Gamma+\Gamma_{c}\right) u(k T)^{+}
\end{aligned}
$$

As stated in Lemma 3.1, the product of matrices $\Phi$ and $\Phi_{c}$ is commutative, and thus

$$
\Phi_{c} \Phi \Phi_{c}^{-1}=\Phi \Phi_{c} \Phi_{c}^{-1}=\Phi
$$

Therefore, from (16) and (15), it follows that

$$
\begin{aligned}
\dot{x}((k+1) T)= & \Phi \dot{x}(k T)-\Phi \Gamma_{c} u((k-1) T)^{+} \\
& +\left(\Phi_{c} \Gamma+\Gamma_{c}\right) u(k T)^{+}
\end{aligned}
$$

On the other hand, in view of (5) and (6) one can write

$$
\begin{aligned}
\Phi_{c} \Gamma & =\Phi_{c}\left(\sum_{j=0}^{\infty}\left(\Phi_{c}\right)^{j} \frac{T^{(j+1)}}{(j+1) !} \Gamma_{c}\right) \\
& =\left(\sum_{j=0}^{\infty}\left(\Phi_{c}\right)^{(j+1)} \frac{T^{(j+1)}}{(j+1) !}\right) \Gamma_{c}=\left(\sum_{j=1}^{\infty}\left(\Phi_{c}\right)^{j} \frac{T^{j}}{j !}\right) \Gamma_{c} \\
& =(\Phi-I) \Gamma_{c}=\Phi \Gamma_{c}-\Gamma_{c}
\end{aligned}
$$


and thus, from (17) and (18):

$$
\begin{aligned}
\dot{x}((k+1) T)= & \Phi \dot{x}(k T)-\Phi \Gamma_{c} u((k-1) T)^{+} \\
& +\Phi \Gamma_{c} u(k T)^{+}
\end{aligned}
$$

Finally, by using $\xi(k T)$ defined in (9), Equation (19) can be rewritten as (8) with the $A, B$ matrices given in (10).

Remark 3.1: The representation (8) derived in Theorem 1 can be employed to design control laws of the form:

$$
u(k T)^{+}=F \xi(k T)
$$

where $F \in \mathbb{R}^{m \times(n+m)}$ is a feedback gain matrix. It is worth noting that (20) can be regarded as a dynamic state derivative feedback expression, because $\xi(k T)$ includes the past control value $u((k-1) T)^{+}$. However, the design of the gain matrix $F$ can be carried out through standard discrete time state space methods and does not require the choice of a particular structure for the controller. In the examples presented in Section 4, it is assumed that the control task starts at time $k=0$ and thus the control law is initialised with $u(-T)^{+}=0$.

Remark 3.2 (Uncertain time delay at the plant input): Consider a model of the form (1) with known matrices $\Phi_{c}, \Gamma_{c}$ and an uncertain time delay $\tau$, i.e.

$$
\dot{x}(t)=\Phi_{c} x(t)+\Gamma_{c} u(t-\tau)
$$

For simplicity, assume that $\tau=d T$, where $d$ is an uncertain integer value in a given range $[0, \bar{d}]$. Therefore, (21) can be discretised as $x((k+1) T)=\Phi x(k T)+\Gamma u((k$ $-d) T$ ), with $\Phi$ and $\Gamma$ as in (5) and (6), respectively. Theorem 1 can then be used to obtain the following discrete time state derivative model:

$$
\begin{aligned}
\dot{x}((k+1) T)= & \Phi \dot{x}(k T)-\Phi \Gamma_{c} u((k-d-1) T)^{+} \\
& +\Phi \Gamma_{c} u((k-d) T)^{+}
\end{aligned}
$$

which can be recast into the form of a polytopic model, with vertices associated to the possible values of $d$ (Huang \& Wang, 2009; Rossi \& Galvão, 2014). The case of different delays for each input variable can be treated in a similar manner, with vertices associated to the possible delay combinations. Uncertainties in both the time delay and the model matrices $\Phi_{c}, \Gamma_{c}$ can be handled through the use of more elaborate formulations, such as the method proposed in Ding, Xie, and Cai (2008) for the case of state feedback.

Remark 3.3 (Polytopic uncertainties): Consider a model of the form (1), with matrices $\Phi_{c}$ and $\Gamma_{c}$ subject to polytopic uncertainties, i.e. $\left(\Phi_{c}, \Gamma_{c}\right) \in \Omega_{\Phi_{c}, \Gamma_{c}}$, where $\Omega_{\Phi_{c}, \Gamma_{c}}$ is a polytope with known vertices $\left(\Phi_{c, i}, \Gamma_{c, i}\right)$, $i=1,2, \ldots, N$. Moreover, let $\Phi_{i}=e^{\Phi_{c, i} T}$ and assume that the sampling period $T$ is sufficiently small so that the quadratic and higher order terms in the power series expansion of $e^{\Phi_{c, i} T}$ can be neglected in the uncertainty representation (Colombo Junior, Afonso, Galvão, \& Assunção, 2016). Matrices $\left(\Phi, \Gamma_{c}\right)$ will then lie in a polytope $\Omega_{\Phi, \Gamma_{c}}$ with vertices $\left(\Phi_{i}, \Gamma_{c, i}\right), i=1,2, \ldots, N$. Finally, in view of the product between $\Phi$ and $\Gamma_{c}$ in (10), the $(A$, $B$ ) matrices in (8) will belong to a polytope with $N^{2}$ vertices, which are associated to the cross-products between the vertices $\Phi_{i}, i=1,2, \ldots, N$, and $\Gamma_{c, j}, j=1,2, \ldots$, $N$. An alternative uncertainty description for matrix $\Phi$ could also be derived by taking into account higher order terms in the power series expansion of $e^{\Phi_{c} T}$, as proposed in Braga, Morais, Tognetti, Oliveira, and Peres (2013). However, the approach described herein leads to simpler design procedures and can be appropriate to meet closed loop specifications, as will be illustrated in Section 4.3.

\section{Examples}

This section presents three computational examples of control design employing the discrete time state derivative representation (8) derived in Theorem 1.

\subsection{Example 1: Discrete time linear quadratic regulator}

This first example illustrates the convenience of taking into account the effect of sampling when designing a control law with state derivative feedback for discrete time implementation. For this purpose, the proposed representation (8) derived in Theorem 1 will be used to design a discrete time linear quadratic regulator (DLQR), which will be compared with a LQR designed in continuous time (Duan et al., 2005; Tseng \& Hsieh, 2013) and emulated in discrete time.

The plant under consideration is a vibration suppression system, consisting of two masses $m_{1}$ and $m_{2}$ coupled by a spring-damper device (stiffness $k_{2}$ and damping $b_{2}$ ), with an additional spring and damper between $m_{1}$ and the ground (stiffness $k_{1}$ and damping $b_{1}$ ), as described by Abdelaziz and Valášek (2004). The plant dynamics can be represented by a continuous time state equation of the form (1) with the following state vector $x(t)$ and model matrices $\Phi_{c}, \Gamma_{c}$ (Abdelaziz \& Valášek, 2004):

$$
\begin{aligned}
x(t) & =\left[\begin{array}{c}
x_{1}(t) \\
x_{2}(t) \\
\dot{x}_{1}(t) \\
\dot{x}_{2}(t)
\end{array}\right], \quad \Phi_{c}=\left[\begin{array}{cccc}
0 & 0 & 1 & 0 \\
0 & 0 & 0 & 1 \\
\frac{-k_{1}-k_{2}}{m_{1}} & \frac{k_{2}}{m_{1}} & \frac{-b_{1}-b_{2}}{m_{1}} & \frac{b_{2}}{m_{1}} \\
\frac{k_{2}}{m_{2}} & \frac{-k_{2}}{m_{2}} & \frac{b_{2}}{m_{2}} & \frac{-b_{2}}{m_{2}}
\end{array}\right], \\
\Gamma_{c} & =\left[\begin{array}{c}
0 \\
0 \\
-\frac{1}{m_{1}} \\
\frac{1}{m_{2}}
\end{array}\right]
\end{aligned}
$$



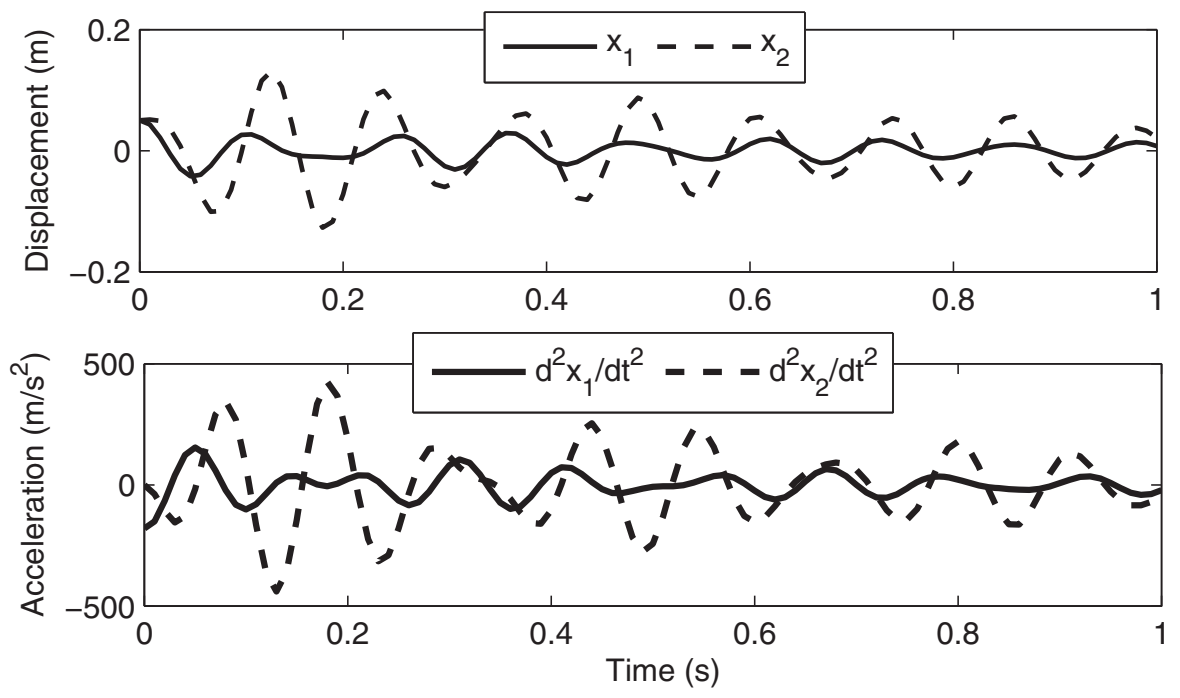

Figure 2. Open-loop response of the vibration suppression system.

where $x_{1}$ and $x_{2}$ denote the vertical displacements of masses $m_{1}$ and $m_{2}$, respectively, and $\dot{x}_{1}$ and $\dot{x}_{2}$ denote the corresponding velocities. The control input $u$ is the force provided by an actuator between the two masses. The model parameters adopted herein were $m_{1}=100 \mathrm{~kg}$, $m_{2}=10 \mathrm{~kg}, k_{1}=360 \mathrm{kN} / \mathrm{m}, k_{2}=36 \mathrm{kN} / \mathrm{m}, b_{1}=70 \mathrm{Ns} / \mathrm{m}$ and $b_{2}=50 \mathrm{Ns} / \mathrm{m}$, as in Abdelaziz and Valášek (2004). For illustration, Figure 2 presents the open loop response, starting from the initial condition $x(0)=[0.05 \mathrm{~m} 0.05 \mathrm{~m}$ $0.2 \mathrm{~m} / \mathrm{s} 0.2 \mathrm{~m} / \mathrm{s}]^{T}$.

The problem consists of designing a feedback control law employing the state derivative $\dot{x}$, which comprises the vertical velocities $\dot{x}_{1}, \dot{x}_{2}$ and accelerations $\ddot{x}_{1}, \ddot{x}_{2}$.

A continuous time LQR controller can be designed by minimising a quadratic cost function $J_{l q r}$ of the form

$$
J_{l q r}=\int_{0}^{\infty}\left[\|\dot{x}(t)\|_{S}^{2}+\|u(t)\|_{R}^{2}\right] d t
$$

where $S \in \mathbb{R}^{n \times n}, R \in \mathbb{R}^{m \times m}$ are positive definite weight matrices and $\dot{x}(t), u(t)$ are related through the continuous time model (1). It is assumed that matrix $\Phi_{c}$ is nonsingular, as discussed in Remark 2.1. Under this assumption, let $G=\Phi_{c}^{-1}$ and $H=-\Phi_{c}^{-1} \Gamma_{c}$. The optimal control is then given by $u(t)=F_{l q r} \dot{x}(t)$ with gain $F_{l q r}$ calculated as

$$
F_{l q r}=-R^{-1} H^{T} P
$$

where $P$ is the positive definite solution of the following Riccati equation:

$$
P G+G^{T} P-P H R^{-1} H^{T} P+S=0
$$

Details regarding the solution of this state derivative LQR problem can be found, for instance, in Duan et al. (2005); Tseng and Hsieh (2013).

In this example, the cost function weights were set to $S$ $=\operatorname{diag}(1,1,1,1)$ and $R=0.02$. The Matlab ${ }^{\circledR}$ Control System Toolbox ${ }^{\mathrm{TM}}$ was employed to solve the Riccati equation (26) and calculate the gain matrix $F_{l q r}$ as in (25), with the following result:

$$
F_{l q r}=[199.6-363.9-0.76-2.34]
$$

For discrete time implementation, the $\mathrm{LQR}$ controller was emulated by making

$$
u(k T)^{+}=F_{l q r} \dot{x}(k T)
$$

and keeping the control $u(t)$ constant between sampling times, as in (2). It is worth noting that the value of the sampling period $T$ was not taken into account in the calculation of $F_{l q r}$, since the design was based on a continuous time formulation.

Figure 3 presents the closed-loop responses of the displacements $x_{1}, x_{2}$, accelerations $\ddot{x}_{1}, \ddot{x}_{2}$ and control input $u$, with two different sampling periods $(T=0.01 \mathrm{~s}$ in Figure 3(a) and $T=0.04 \mathrm{~s}$ in Figure 3(b)). The displacements are shown for better clarity, but it is worth recalling that the control law employs the velocities and accelerations. As can be seen in Figure 3(a), the LQR control law emulated with $T=0.01 \mathrm{~s}$ improves the damping of the oscillations compared to the open-loop response in Figure 2. On the other hand, for $T=0.04 \mathrm{~s}$ (Figure 3(b)), the closed-loop system becomes unstable. This result can be ascribed to the effect of sampling, which was not taken into account in the LQR design. 
(a) $T=0.01 \mathrm{~s}$
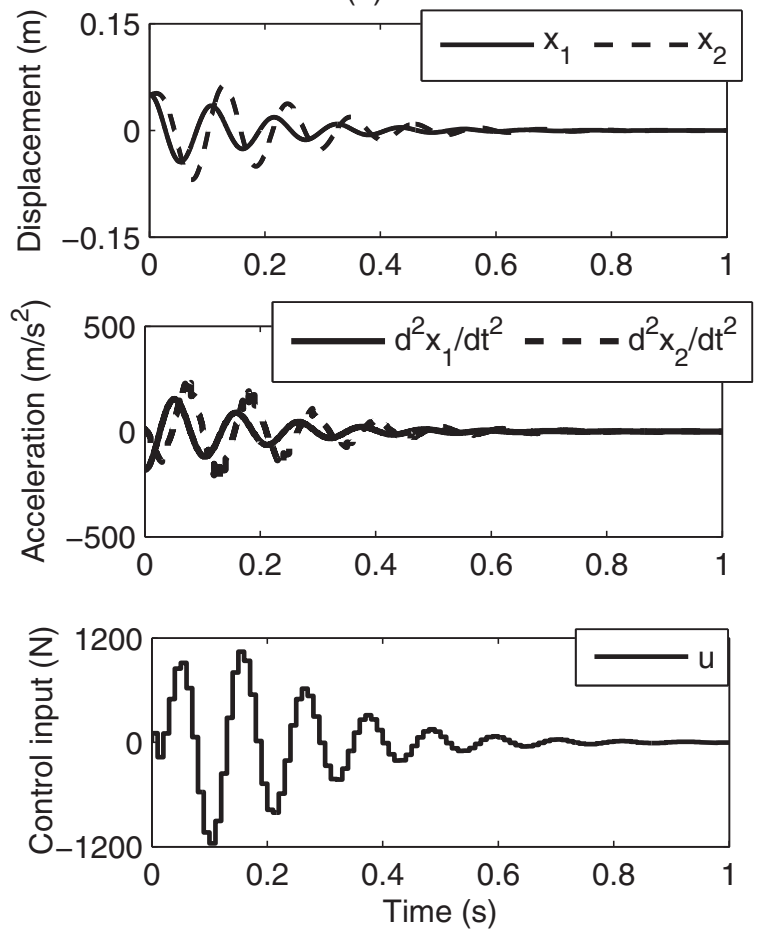

(b) $T=0.04 \mathrm{~s}$
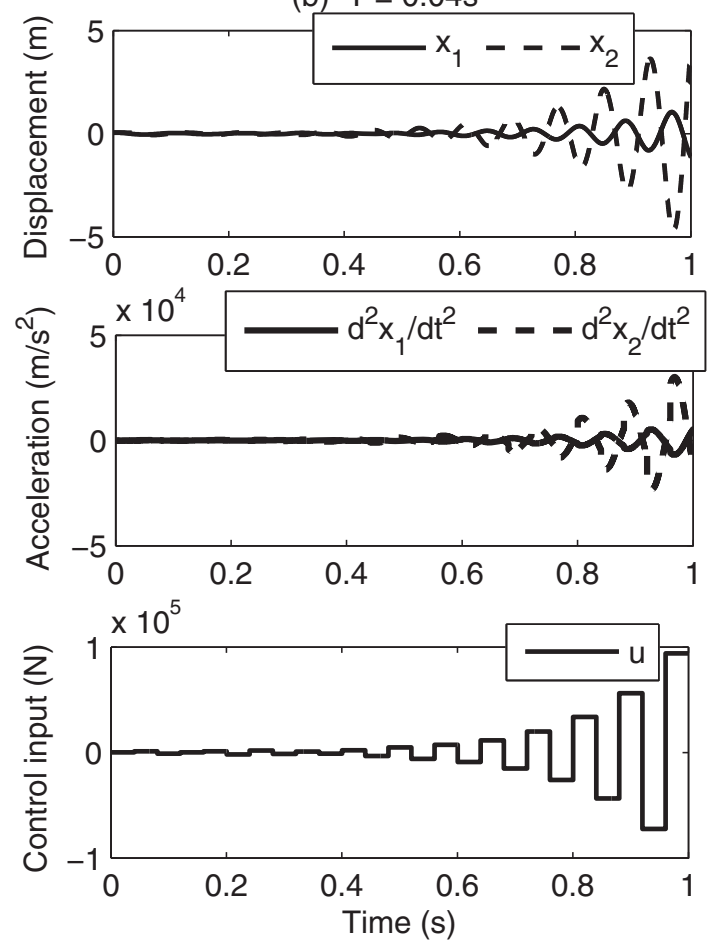

Figure 3. Closed-loop responses: LQR emulated with sampling period (a) $T=0.01 \mathrm{~s}$ and (b) $T=0.04 \mathrm{~s}$.

In order to account for the effect of sampling, a DLQR controller can be designed by using the proposed discrete time state derivative model (8), with $\xi(k T)$ given by

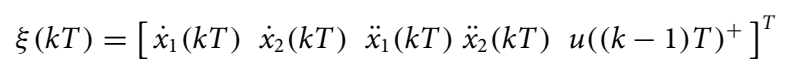

and $A, B$ as in (10). In this case, the problem consists of minimising a quadratic cost function $J_{d l q r}$ of the form

$$
J_{d l q r}=\sum_{k=0}^{\infty}\left[\|\xi(k T)\|_{S}^{2}+\left\|u(k T)^{+}\right\|_{R}^{2}\right]
$$

with positive definite weight matrices $S, R$. The optimal control is given by $u(k T)^{+}=F_{d l q r} \xi(k T)$, with gain $F_{d l q r}$ calculated as (Lewis \& Syrmos, 1995):

$$
F_{d l q r}=-\left(B^{T} P B+R\right)^{-1}\left(B^{T} P A\right)
$$

where $P$ is the positive definite solution of the following Riccati equation:

$$
A^{T} P A-P-\left(A^{T} P B\right)\left(B^{T} P B+R\right)^{-1}\left(B^{T} P A\right)+S=(B 2)
$$

For comparison with the LQR results presented above, the DLQR cost function weights were set to $S=\operatorname{diag}(1,1$, $1,1,0.01)$ and $R=0.01$. Since the augmented state vector $\xi(k T)$ in (29) includes the past control value $u((k-1) T)^{+}$, the control weight 0.02 adopted in the LQR design was equally divided into $R$ and the last diagonal element of $S$.

It is worth noting that the $A, B$ model matrices employed in the DLQR design depend on the sampling period $T$. Therefore, different DLQR gain matrices were obtained for $T=0.01 \mathrm{~s}$ and $T=0.04 \mathrm{~s}$. The Matlab ${ }^{\circledR}$ Control System Toolbox ${ }^{\mathrm{TM}}$ was employed to solve the Riccati equation (32) and calculate the gain matrix $F_{d l q r}$ as in (31). The results obtained for $T=0.01 \mathrm{~s}$ and $T=0.04 \mathrm{~s}$ are presented in (33) and (34), respectively:

$$
\begin{aligned}
& F_{\text {dlqr }(T=0.01 s)}=\left[\begin{array}{lllll}
101.8 & -221.6 & -0.074 & -2.70 & 0.27
\end{array}\right] \\
& F_{d l q r(T=0.04 s)}=\left[\begin{array}{lllll}
71.6 & -108.7 & -0.29 & -3.33 & 0.33
\end{array}\right]
\end{aligned}
$$

The resulting closed-loop responses with $T=0.01 \mathrm{~s}$ as shown in Figure 4(a) and $T=0.04 \mathrm{~s}$ as shown in Figure 4(b). As can be seen by comparing Figure 3(a) and Figure 4(a), the LQR and DLQR results are similar for $T=$ $0.01 \mathrm{~s}$. On the other hand, the DLQR controller preserves the stability of the system for $T=0.04 \mathrm{~s}$ (Figure 4(b)), whereas the emulated LQR does not (Figure 3(b)).

It is also worth noting that the proposed discrete time model (8) could be employed to predict the unstable behaviour resulting from the use of the emulated LQR 
(a) $T=0.01 \mathrm{~s}$
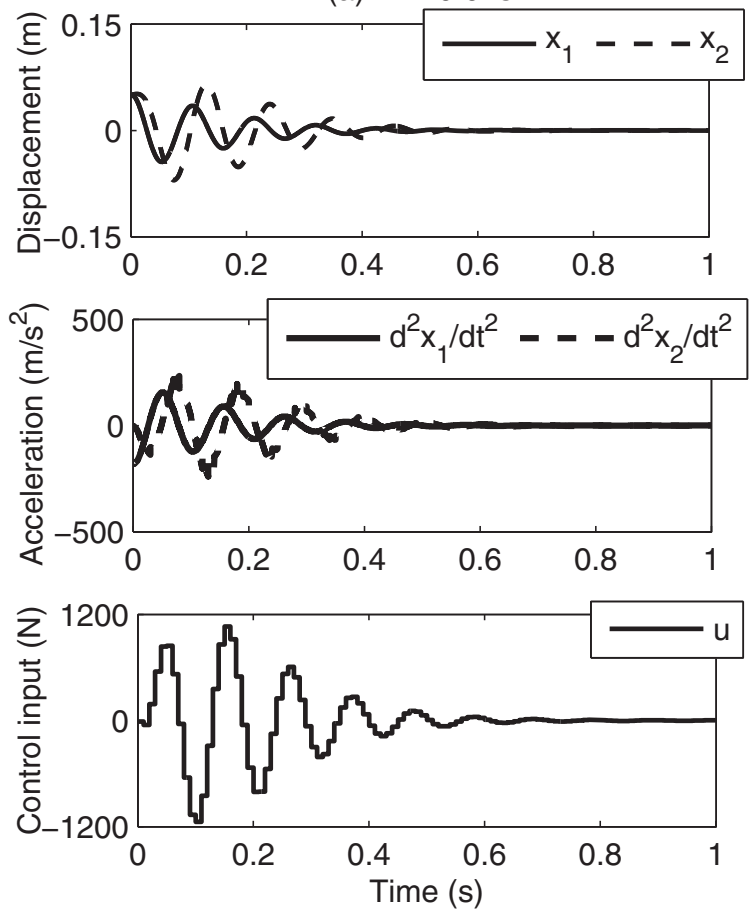

(b) $T=0.04 \mathrm{~s}$
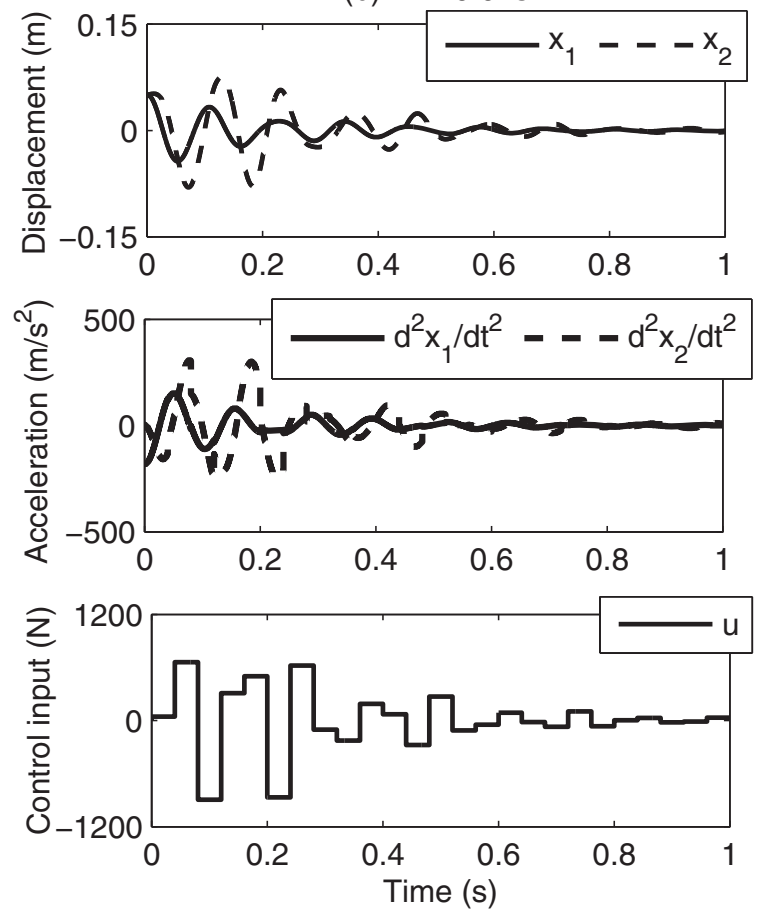

Figure 4. Closed-loop responses: DLQR with sampling period (a) $T=0.01 \mathrm{~s}$ and (b) $T=0.04 \mathrm{~s}$.

with $T=0.04 \mathrm{~s}$. In fact, in view of (8), (9), (28), the closedloop dynamics of the sampled system can be described by a state equation of the form $\xi((k+1) T)=(A+$ $\left.B \bar{F}_{l q r}\right) \xi(k T)$, where

$$
\bar{F}_{l q r}=\left[\begin{array}{c}
F_{l q r} \\
0
\end{array}\right]
$$

Figure 5 presents the closed-loop poles obtained with the DLQR and emulated LQR control laws, which correspond to the eigenvalues of $A+B F_{d l q r}$ and $A+B \bar{F}_{l q r}$, respectively. As shown in Figure 5(a), the two control laws result in similar closed-loop poles for $T=0.01 \mathrm{~s}$, which is in agreement with the similar responses in Figure 3(a) and Figure 4(a). However, by increasing the sampling period to $T=0.04 \mathrm{~s}$, one of the closed-loop poles obtained with the emulated LQR controller leaves the unit circle in Figure 5(b), which explains the unstable behaviour seen in Figure 3(b). In contrast, the closed-loop poles obtained with the DLQR formulation remain inside the unit circle

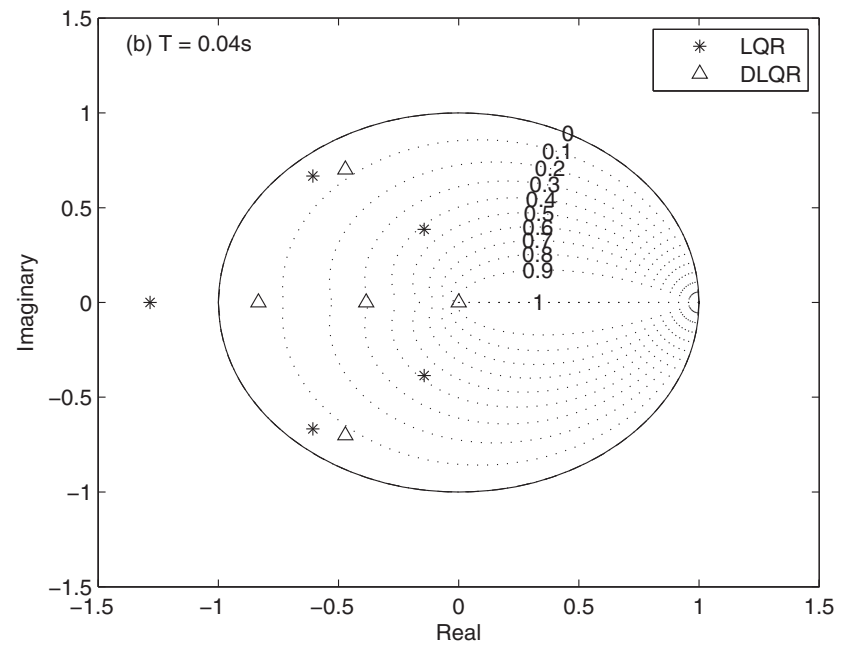

Figure 5. Closed-loop poles obtained with the DLQR and emulated LQR controllers using (a) $T=0.01 \mathrm{~s}$ and (b) $T=0.04 \mathrm{~s}$. 
(a) Without input constraints
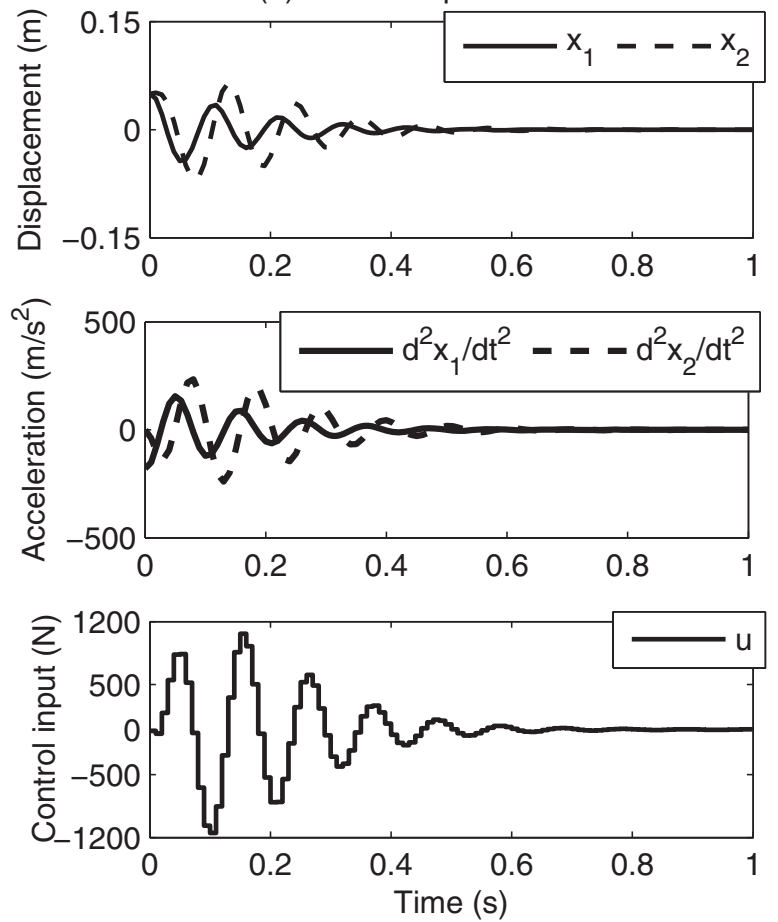

(b) With input constraints
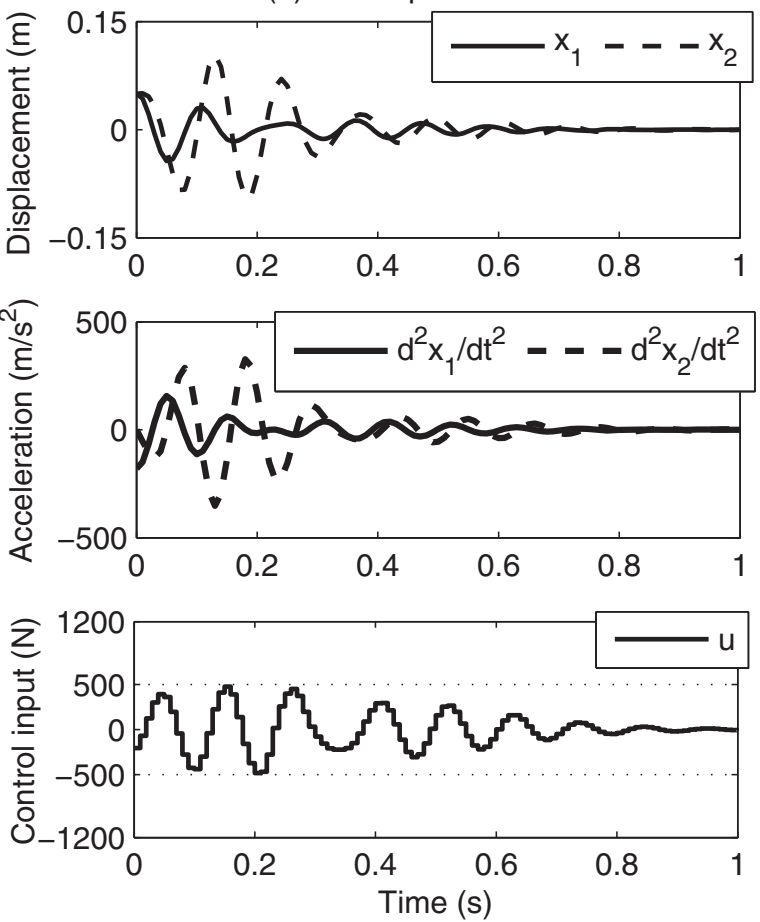

Figure 6. Closed-loop responses: NMPC controller (a) without and (b) with the imposition of input constraints.

in Figure 5(b), and thus stability is preserved, as seen in Figure 4(b).

\subsection{Example 2: Robust model predictive control}

This second example illustrates the use of discrete time state derivative feedback within a robust model predictive control (RMPC) framework. More specifically, the classic LMI-based RMPC formulation proposed by Kothare, Balakrishnan, and Morari (1996) is employed (see Appendix 1). In all the simulations, the LMI tools of the Matlab ${ }^{\circledR}$ Robust Control Toolbox ${ }^{\mathrm{TM}}$ were used to solve the semidefinite programming problem involved in this formulation. The plant under consideration is the same vibration suppression system described in Section 4.1 , with a sampling period $T=0.01 \mathrm{~s}$ and initial condition set to $x(0)=[0.05 \mathrm{~m} 0.05 \mathrm{~m} 0.2 \mathrm{~m} / \mathrm{s} 0.2 \mathrm{~m} / \mathrm{s}]^{T}$.

In what follows, a predictive controller will be initially designed for the nominal case, in which there are no uncertainties in the plant model. This case will be employed to illustrate the imposition of constraints on the control amplitude. The design of the controller will then be extended to account for an uncertain time delay at the plant input, in addition to the control constraints.

As discussed in Section 4.1, the discrete time state derivative model for the plant can be written as $\xi((k+$ $1) T)=A \xi(k T)+B u(k T)^{+}$, with $\xi(k T)$ given by (29). The formulation presented in Appendix 1 can then be used with $\xi(k T), A, B$ in place of $x(k T), \Phi, \Gamma$, respectively. In this case, a single vertex is employed in the LMIs. The cost function weights were set to $S=\operatorname{diag}(1$, $1,1,1,0.01$ ) and $R=0.01$. Henceforth, this control law will be termed NMPC (Nominal Model Predictive Control).

As can be seen in Figure 6(a), the resulting closed-loop response is identical to the response obtained with the DLQR controller (Figure 4(a)). Indeed, as discussed in Kothare et al. (1996), in the absence of constraints and model uncertainty, the LMI-based predictive control formulation is equivalent to a DLQR control law with the same cost weights. However, in the general case (involving constraints and/or model uncertainty) the predictive control law will be nonlinear, because a new gain matrix $F$ is calculated at each sampling time on the basis of the feedback measurements.

To illustrate the imposition of control constraints, the simulation was repeated after including control bounds of $\pm 500 \mathrm{~N}$ in the NMPC formulation. The results are presented in Figure 6(b), with horizontal dotted lines indicating the bounds on the control input. As can be seen, the constraints were properly enforced, at the cost of a slight increase in the amplitude of the displacements and accelerations. It is worth noting that the control constraints were satisfied without the need to modify the cost function weights. This simplification in the design procedure is an advantage of the predictive control approach over 


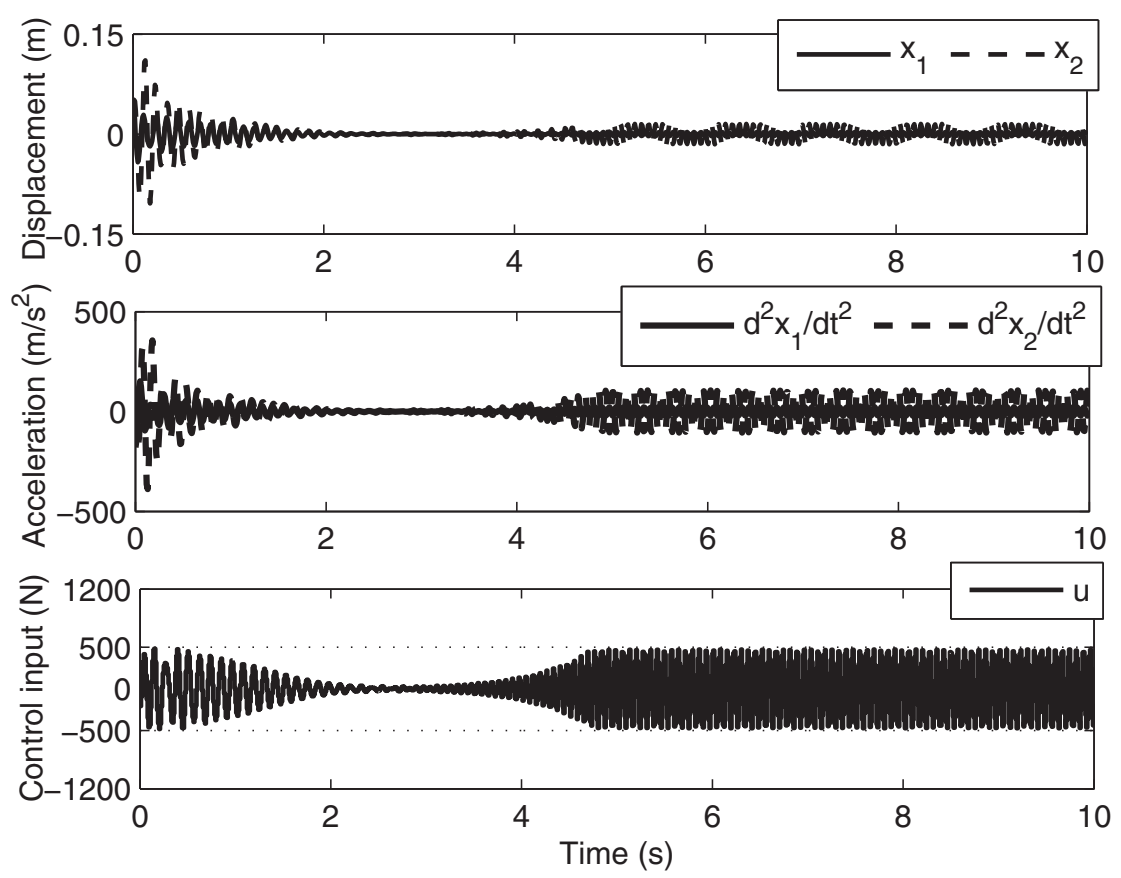

Figure 7. Closed-loop response: NMPC controller, plant with input time delay $(d=2)$.

the DLQR formulation regarding the control of systems with input constraints.

It will be now assumed that the plant input is subject to an uncertain time delay of up to $\bar{d}=2$ sampling periods. The input delay has a destabilising effect in the NMPC control loop, as shown in Figure 7, which justifies the design of an RMPC controller.

For this purpose, the delay uncertainty can be handled as described in Remark 3.2, by casting the discrete time model into the form $v((k+1) T)=A_{v} v(k T)+B_{v} u(k T)^{+}$, with $v(k T)$ given by

$v(k T)=\left[\dot{x}^{T}(k T) u((k-1) T)^{+} u((k-2) T)^{+} u((k-3) T)^{+}\right]^{T}$

and

$$
\begin{aligned}
A_{v} & =\left[\begin{array}{cccc}
\Phi\left(\lambda_{1}-\lambda_{0}\right) \Phi \Gamma_{c} & \left(\lambda_{2}-\lambda_{1}\right) \Phi \Gamma_{c}-\lambda_{2} \Phi \Gamma_{c} \\
0 & 0 & 0 & 0 \\
0 & 1 & 0 & 0 \\
0 & 0 & 1 & 0
\end{array}\right] \\
B_{v} & =\left[\begin{array}{c}
\lambda_{0} \Phi \Gamma_{c} \\
1 \\
0 \\
0
\end{array}\right]
\end{aligned}
$$

In this case, $\left(A_{v}, B_{v}\right)$ belongs to a polytope $\Omega_{v}$ with three vertices $\left(A_{v 1}, B_{v 1}\right),\left(A_{v 2}, B_{v 2}\right),\left(A_{v 3}, B_{v 3}\right)$, which correspond to the values $(1,0,0),(0,1,0)$ and $(0,0,1)$ for $\left(\lambda_{0}, \lambda_{1}, \lambda_{2}\right)$ in (37). This uncertain model can be used in the RMPC formulation presented in Appendix 1, with $v(k T), A_{v}, B_{v}$ in place of $x(k T), \Phi, \Gamma$, respectively. Following the rationale discussed in Section 4.1 regarding the division of the control weights into the $S$ and $R$ matrices, the RMPC controller was implemented with $S=\operatorname{diag}(1$, $1,1,1,0.005,0.005,0.005)$ and $R=0.005$.

As can be seen in Figure 8, by using the RMPC formulation, similar results are obtained in the absence (Figure 8(a)) or in the presence (Figure 8(b)) of the input delay, which corroborates the robustness of the controller. It is worth noting that the control amplitude constraint is properly enforced in both cases, as shown in the bottom plots of Figure 8.

\subsection{Example 3: Regional pole placement}

This third example illustrates the use of the proposed representation (8) derived in Theorem 1 for discrete time design of a state derivative feedback control law in the presence of parametric uncertainties. For this purpose, the classic LMI approach for regional pole placement proposed by Chilali and Gahinet (1996) is employed (see Appendix 2).

The plant under consideration is an active suspension system for a car seat, with a car mass $M_{c}$ and a seat-plusdriver mass $m_{s}$, as described by Faria et al. (2009). Passive shock absorbers in the car body (stiffness $k_{1}$ and damping $b_{1}$ ) and driver seat (stiffness $k_{2}$ and damping $b_{2}$ ) provide partial attenuation of vertical vibrations caused by irregularities on the ground. The control variables consist of two 
(a) Plant without input time delay
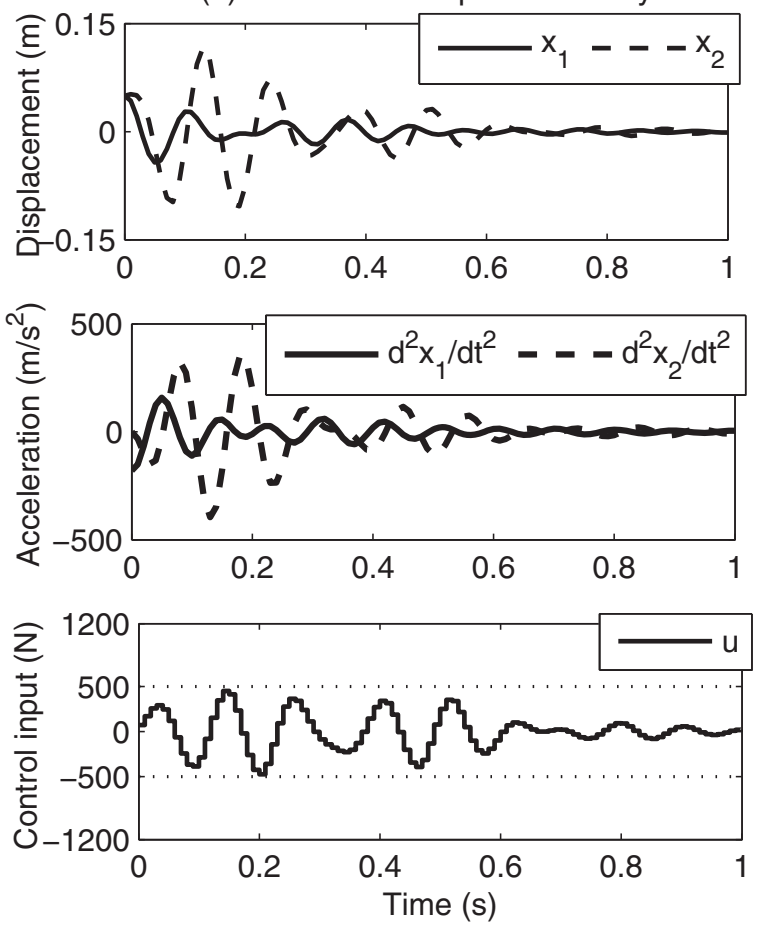

(b) Plant with input time delay $(\mathrm{d}=2)$
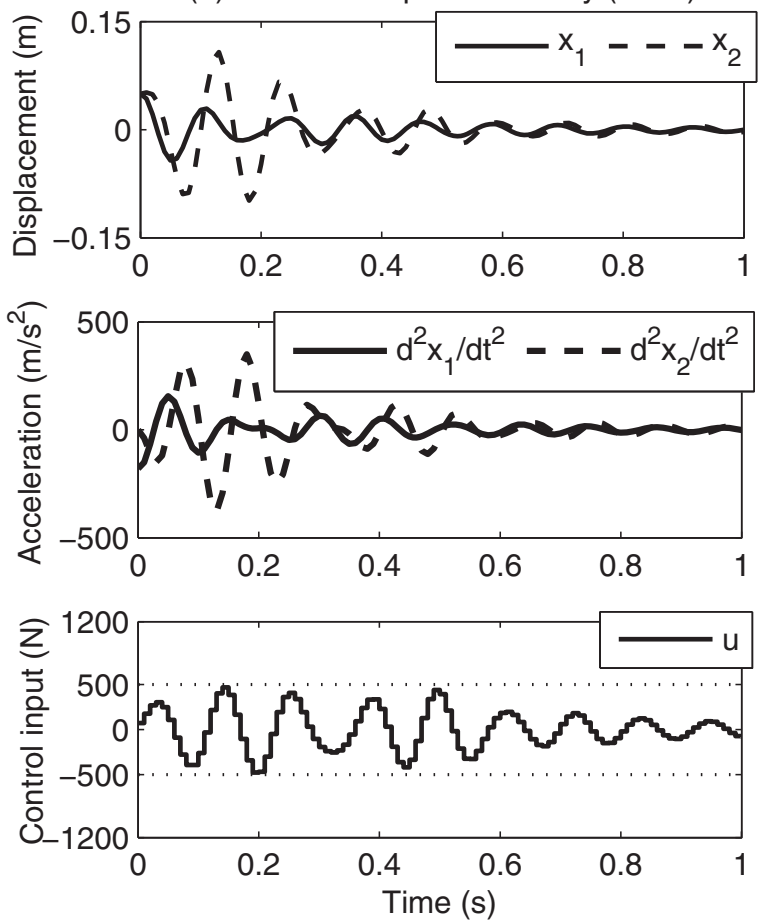

Figure 8. Closed-loop responses: RMPC controller, (a) plant without and (b) with input time delay $d=2$.

forces $u_{1}$ and $u_{2}$, which are generated by actuators at the suspension mechanisms of the car and seat, respectively.

The plant dynamics can be described by a continuous time state equation of the form (1) with the following state vector $x(t)$ and model matrices $\Phi_{c}, \Gamma_{c}$ (Faria et al., 2009):

$$
\begin{aligned}
x(t)= & {\left[\begin{array}{c}
x_{1}(t) \\
x_{2}(t) \\
\dot{x}_{1}(t) \\
\dot{x}_{2}(t)
\end{array}\right], \quad \Phi_{c}=\left[\begin{array}{cccc}
0 & 0 & 1 & 0 \\
0 & 0 & 0 & 1 \\
\frac{-k_{1}-k_{2}}{M_{c}} & \frac{k_{2}}{M_{c}} & \frac{-b_{1}-b_{2}}{M_{c}} & \frac{b_{2}}{M_{c}} \\
\frac{k_{2}}{m_{s}} & \frac{-k_{2}}{m_{s}} & \frac{b_{2}}{m_{s}} & \frac{-b_{2}}{m_{s}}
\end{array}\right] } \\
\Gamma_{c}= & {\left[\begin{array}{cc}
0 & 0 \\
0 & 0 \\
-\frac{1}{M_{c}} & -\frac{1}{M_{c}} \\
0 & \frac{1}{m_{s}}
\end{array}\right] }
\end{aligned}
$$

where $x_{1}$ and $x_{2}$ denote the vertical displacements of masses $M_{c}$ and $m_{s}$, respectively, and $\dot{x}_{1}$ and $\dot{x}_{2}$ represent the corresponding velocities. The model parameters were adopted as in Faria et al. (2009): $M_{c}=1500 \mathrm{~kg}, 70 \mathrm{~kg} \leq$ $m_{s} \leq 120 \mathrm{~kg}$ (assuming a seat mass of $20 \mathrm{~kg}$ and an uncertain driver mass ranging from 50 to $100 \mathrm{~kg}$ ), $k_{1}=4 \times$ $10^{4} \mathrm{~N} / \mathrm{m}, k_{2}=5 \times 10^{3} \mathrm{~N} / \mathrm{m}, b_{1}=4 \times 10^{3} \mathrm{Ns} / \mathrm{m}$ and $b_{2}=$ $5 \times 10^{3} \mathrm{Ns} / \mathrm{m}$. Therefore, the model is of the form (1) with $\left(\Phi_{c}, \Gamma_{c}\right) \in \operatorname{Co}\left\{\left(\Phi_{c, 1}, \Gamma_{c, 1}\right),\left(\Phi_{c, 2}, \Gamma_{c, 2}\right)\right\}$, where $m_{s}=70$ $\mathrm{kg}$ for $\left(\Phi_{c, 1}, \Gamma_{c, 1}\right)$ and $m_{s}=120 \mathrm{~kg}$ for $\left(\Phi_{c, 2}, \Gamma_{c, 2}\right)$.

The open-loop responses of the system with the extreme mass values $m_{s}=70 \mathrm{~kg}$ as shown in Figure 9(a)
Table 1. Eigenvalues of the continuous time model vertices, with corresponding damping ratios $(\zeta)$, natural frequencies $\left(\omega_{n}\right)$ and natural oscillation periods $T_{n}=2 \pi / \omega_{n}$.

\begin{tabular}{ccccc}
\hline & Eigenvalues & $\zeta$ & $\omega_{n}(\mathrm{rad} / \mathrm{s})$ & $T_{n}(\mathrm{~s})$ \\
\hline$\Phi_{c, 1}$ & $-3.83 \pm j 7.87$ & 0.49 & 8.75 & 0.72 \\
& $-1.24 \pm j 4.83$ & 0.26 & 4.99 & 1.26 \\
$\Phi_{c, 2}$ & $-2.45 \pm j 6.56$ & 0.37 & 7.00 & 0.90 \\
& $-1.13 \pm j 4.63$ & 0.24 & 4.76 & 1.32 \\
\hline
\end{tabular}

and $m_{s}=120 \mathrm{~kg}$ as shown in Figure 9(b). The initial conditions were set to $x(0)=[0.2 \mathrm{~m} 0.1 \mathrm{~m} 0 \mathrm{~m} / \mathrm{s} 0 \mathrm{~m} / \mathrm{s}]^{T}$.

Table 1 presents the eigenvalues of matrices $\Phi_{c, 1}$ and $\Phi_{c, 2}$, as well as the corresponding damping ratios $(\zeta)$, natural frequencies $\left(\omega_{n}\right)$ and natural oscillation periods $T_{n}=2 \pi / \omega_{n}$. As can be seen, the plant has two secondorder modes, with dynamic features that depend on the uncertain driver mass. For discrete time control purposes, the sampling period was chosen as $T=0.10 \mathrm{~s}$, which is approximately 10 times smaller than the smallest $T_{n}$ value in Table 1.

In light of Theorem 1 , the resulting discrete time plant model can be cast into the form $\xi((k+1) T)=A \xi(k T)+$ $B u(k T)^{+}$, with $A, B$ as in (10) and

$$
\xi(k T)=\left[\dot{x}_{1}(k T) \dot{x}_{2}(k T) \ddot{x}_{1}(k T) \ddot{x}_{2}(k T) u((k-1) T)^{+}\right]^{T}
$$


(a) $m_{s}=70 \mathrm{~kg}$
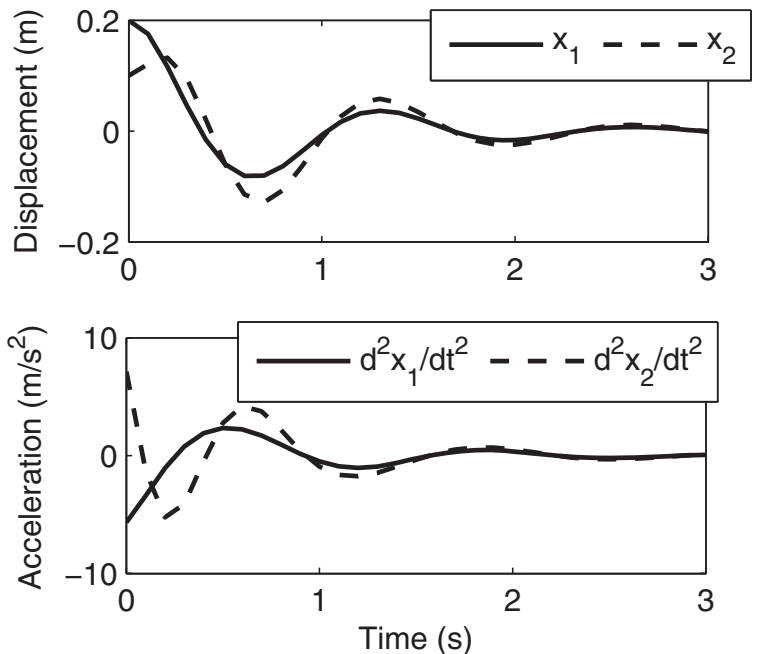

(b) $m_{s}=120 \mathrm{~kg}$
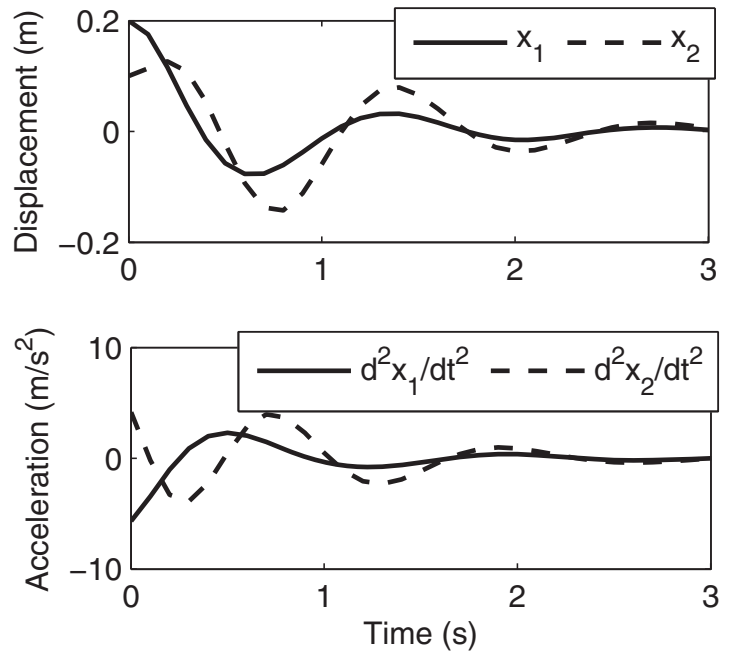

Figure 9. Open-loop responses of the active suspension system with (a) $m_{s}=70 \mathrm{~kg}$ and (b) $m_{s}=120 \mathrm{~kg}$.

Figure 10(a) presents the open-loop poles of the discrete time model (i.e. the eigenvalues of $A$ ), obtained by varying the parameter $m_{s}$ in the range $[70,120] \mathrm{kg}$. It is worth noting that there is a double pole at the origin, which is associated to the two rows of zeros (one for each component of the input vector $u$ ) in the structure of the $A$ matrix defined in (10).

The pole placement problem considered herein consists of designing a control law of the form $u(k T)^{+}=$ $F \xi(k T)$, so that the closed-loop poles are placed in a desired allocation region for any value of the uncertain parameter $m_{s}$. More specifically, in order to improve the transient response of the system, it would be convenient to obtain larger damping ratios $\zeta$ and bring the poles closer to the origin. For this purpose, the allocation region was chosen as a circle of radius $r=0.3$ centred at $(0.4,0)$ (depicted as a thick line in Figure $10 \mathrm{~b}$ ).

The gain matrix $F$ was obtained by using the LMI approach of Chilali and Gahinet (1996) described in Appendix 2, with $\xi(k T), A, B$ in place of $x(k T), \Phi, \Gamma$, respectively. It is worth noting that the uncertainty in the parameter $m_{s}$ affects both $\Phi_{c}$ and $\Gamma_{c}$, as can be seen in (38). Therefore, as discussed in Remark 3.3, the $(A$, $B$ ) matrices belong to a polytope with $N^{2}=4$ vertices formed from the pairwise combinations of $\Phi_{1}, \Phi_{2}$ and $\Gamma_{c, 1}, \Gamma_{c, 2}$. A feasible solution to the LMIs involved in the allocation problem was obtained by using the Robust Control Toolbox ${ }^{\mathrm{TM}}$ function 'feasp', as in Mao and Chu
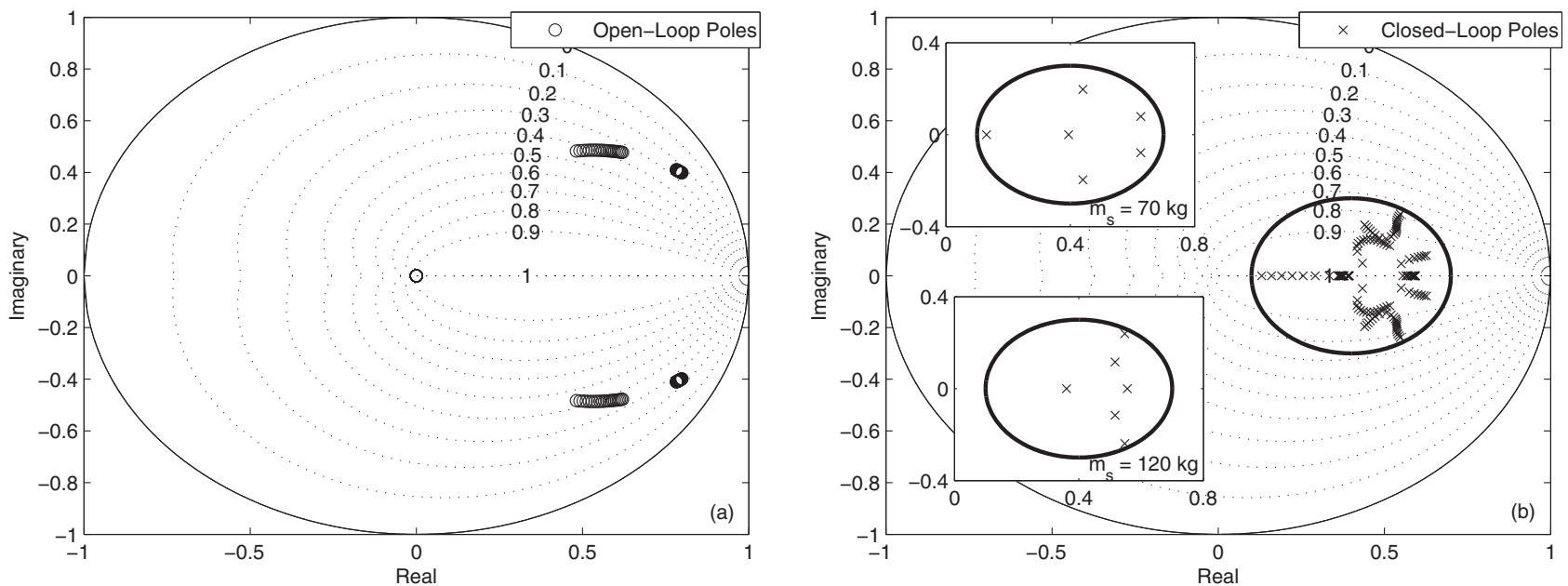

Figure 10. (a) Open-loop poles of the discrete time state derivative model for the active suspension system. The dotted lines correspond to curves of constant damping ratio $\zeta$. (b) Closed-loop poles, with the boundary of the allocation region indicated as a thick line. The closed-loop poles for the extreme values of the uncertain parameter $\left(m_{s}=70 \mathrm{~kg}\right.$ and $\left.m_{\mathrm{s}}=120 \mathrm{~kg}\right)$ are shown as insets. 
(a) $\mathrm{m}_{\mathrm{s}}=70 \mathrm{~kg}$
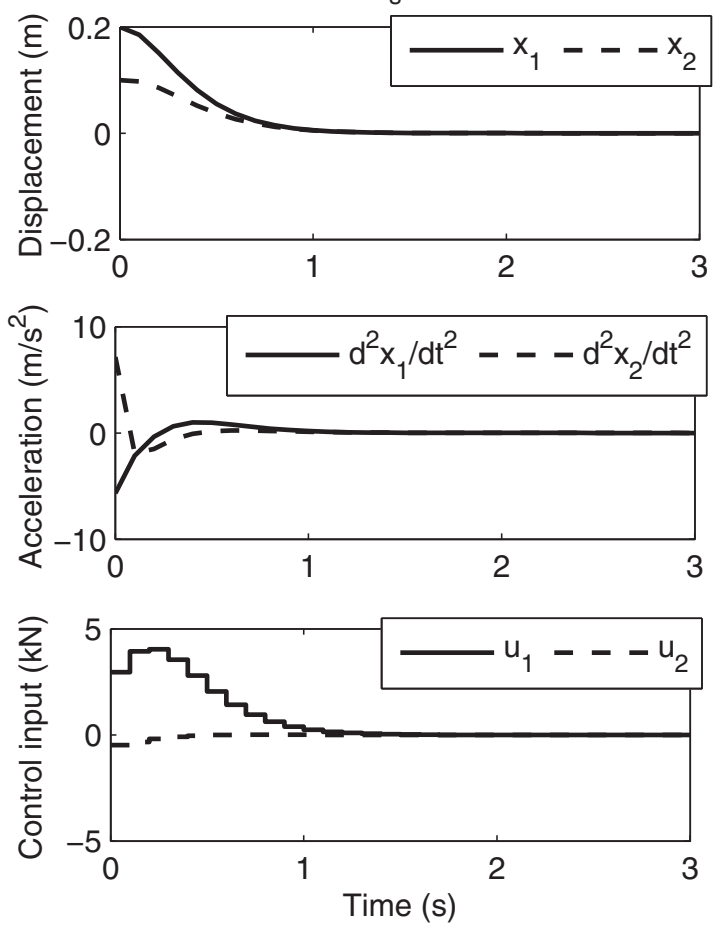

(b) $\mathrm{m}_{\mathrm{s}}=120 \mathrm{~kg}$
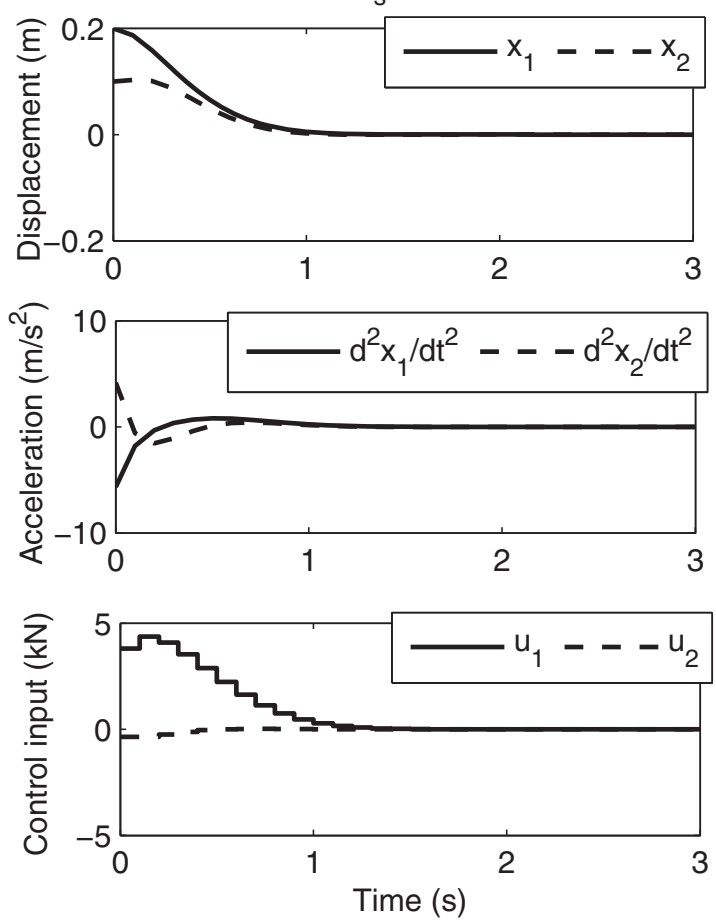

Figure 11. Closed-loop responses for the active suspension system with (a) $m_{s}=70 \mathrm{~kg}$ and (b) $m_{s}=120 \mathrm{~kg}$.

(2009). As a result, the following gain matrix $F$ was obtained:

$F=10^{3} \times\left[\begin{array}{cccccc}-7.3006 & 2.7291 & -0.8821 & -0.2866 & 0.0007 & 0.0047 \\ -0.1593 & -0.2561 & 0.0354 & -0.0387 & -0.0000 & 0.0007\end{array}\right]$

Figure 10(b) shows the closed-loop poles (eigenvalues of $A+B F$ ), again obtained by varying the parameter $m_{s}$ in the range $[70,120] \mathrm{kg}$. The closed-loop poles for the extreme values of the uncertain parameter $\left(m_{s}=70 \mathrm{~kg}\right.$ and $m_{s}=120 \mathrm{~kg}$ ) are presented as insets. As can be seen, all the poles are inside the circle of radius $r=0.3$ centred at $(0.4,0)$ as desired. A comparison with Figure 10(a) reveals that the closed-loop poles are indeed closer to the origin, with larger damping ratios $\zeta$. The closed-loop responses for $m_{s}=70$ as shown in Figure 11(a) and $m_{s}=$ 120 as shown in Figure 11(b). As can be seen, the closedloop system presents better transient responses compared to the open loop system (Figure 9), with suppression of the oscillations and reduction in the settling time.

\section{Conclusion}

This paper presented a method for recasting a continuous time state space model into the form of a discrete time model formulated in terms of the state derivative. Uncertain input delays and polytopic parameter uncertainties in the original state space representation can be propagated to the resulting state derivative model, and thus standard state space techniques can be employed to design robust control laws using discrete time state derivative feedback.

For illustration, three computational examples involving classical design techniques were presented. The first example highlighted the importance of accounting for the effect of sampling when designing a state derivative feedback controller for discrete time implementation, as opposed to the simple emulation of a continuous time control law. More specifically, the example involved the design of LQRs for a vibration suppression system. In this case, the discrete time emulation of the LQR controller resulted in an unstable control loop, whereas the DLQR controller directly designed with the discrete time model derived in Theorem 1 resulted in a stable loop, with better damping compared to the open loop response. In the second example, a RMPC formulation was employed with the same vibration suppression system, in order to handle amplitude constraints and uncertain time delay in the control input. As a result, the closed-loop system was robustly stabilised and the input constraints were properly enforced. This example is of particular interest within the context of robust state derivative feedback, because previous continuous time formulations were not capable of explicitly enforcing constraints on the control amplitude. In the third example, the closed-loop poles of an 
active car seat suspension system were robustly placed inside a desired circle in the complex plane, in the presence of uncertainty in the driver's mass.

Future studies could be concerned with the validation of the proposed formulation in experimental settings.

\section{Disclosure statement}

No conflict of interest is reported by the authors.

\section{Funding}

This work was supported by FAPESP [grant number 2011/17610-0]; CNPq [grant number 140585/2014-1 (Doctoral Scholarship)], [grant number 303714/2014-0], [grant number 310798/2014-0], [grant number 300703/2013-9] (Research Fellowships).

\section{ORCID}

Fernanda Quelho Rossi (1) http://orcid.org/0000-0001-52080792

\section{References}

Abdelaziz, T.H.S. (2009). Robust pole assignment for linear time-invariant systems using state derivative feedback. Proceedings of the Institution of Mechanical Engineers. Part I: Journal of Systems and Control Engineering, 223(2), 187199.

Abdelaziz, T.H.S. (2012). Parametric eigenstructure assignment using state-derivative feedback for linear systems. Journal of Vibration and Control, 18(12), 1809-1827.

Abdelaziz, T.H.S. (2013). Eigenstructure assignment for second-order systems using velocity-plus-acceleration feedback. Structural Control and Health Monitoring, 20(4), 465-482.

Abdelaziz, T.H.S., \& Valášek, M. (2004). Pole-placement for SISO linear systems by state derivative feedback. IEE Proceedings: Control Theory and Applications, 151(4), 377-385.

Amri, I., Soudani, D., \& Benrejeb, M. (2011, March 3-5). Robust state derivative feedback LMI-based designs for time-varying delay system. In Proceeding of the International Conference on Communications, Computing and Control Applications (pp. 1-6). Hammamet: IEEE.

Assunção, E., Teixeira, M.C.M., Faria, F.A., Silva, N.A.P., \& Cardim, R. (2007). Robust state derivative feedback LMIbased designs for multivariable linear systems. International Journal of Control, 80(8), 1260-1270.

Braga, M.F., Morais, C.F., Tognetti, E.S., Oliveira, R.C.L.F., \& Peres, P.L.D. (2013, December 10-13). A new procedure for discretization and state feedback control of uncertain linear systems. In 52nd IEEE Conference on Decision and Control (pp. 6397-6402). Florence: IEEE.

Cardim, R., Teixeira, M.C.M., Faria, F.A., \& Assunção, E. (2009, July 8-10). LMI-based digital redesign of linear time invariant systems with state derivative feedback. In Proceeding of the IEEE Multi-Conference on Systems and Control (pp. 745749). Saint Petersburg: IEEE.
Chilali, M., \& Gahinet, P. (1996). $H_{\infty}$ design with pole placement constraints: An LMI approach. IEEE Trans. Automatic Control, 41(3), 358-367.

Colombo Junior, J.R., Afonso, R.J.M., Galvão, R.K.H., \& Assunção, E. (2016). Robust model predictive control of a benchmark electromechanical system. Journal of Control, Automation and Electrical Systems, 27(2), 119-131.

Ding, B., Xie, L., \& Cai, W. (2008). Robust MPC for polytopic uncertain systems with time-varying delays. International Journal of Control, 81(8), 1239-1252.

Duan, G.-R., \& Yu, H.-H. (2008). Robust pole assignment in high-order descriptor linear systems via proportional plus derivative state feedback. IET Control Theory \& Applications, 2(4), 277-287.

Duan, Y.F., Ni, Y.Q. and Ko, J.M. (2005). State-derivative feedback control of cable vibration using semiactive magnetorheological dampers. Computer-Aided Civil and Infrastructure Engineering, 20(6), 431-449.

Fallah, S., Khajepour, A., Fidan, B., Chen, S. -K., \& Litkouhi, B. (2013). Vehicle optimal torque vectoring using state derivative feedback and linear matrix inequalities. IEEE Transactions on Vehicular Technology, 62(4), 1540-1552.

Faria, F.A., Assunção, E., Teixeira, M.C.M., \& Cardim, R. (2010). Robust state derivative feedback LMI-based designs for linear descriptor systems. Mathematical Problems in Engineering, 2010, 927362.

Faria, F.A., Assunção, E., Teixeira, M.C.M., Cardim, R., \& Silva, N.A.P. (2009). Robust state derivative pole placement LMIbased designs for linear systems. International Journal of Control, 82(1), 1-12.

Franklin, G.F., Powell, J.D., \& Workman, M.L. (1998). Digital control of dynamic systems (3rd ed. ). Menlo Park, CA: Addison Wesley.

Huang, G., \& Wang, S. (2009). Use of uncertainty polytope to describe constraint processes with uncertain time-delay for robust model predictive control applications. ISA Transactions, 48(4), 503-511.

Jing, Y., Shen, C., Dimirovski, G.M., \& Jiang, N. (2009, June 1012). Delay-dependent state derivative feedback with an $\alpha$ stability constraint for time delay systems. In Proceeding of the American Control Conference (pp. 1844-1848). St. Louis, MO.

Kothare, M.V., Balakrishnan, V., \& Morari, M. (1996). Robust constrained model predictive control using linear matrix inequalities. Automatica, 32(10), 1361-1379.

Kwak, S.K., Washington, G., \& Yedavalli, R.K. (2002). Acceleration feedback-based active and passive vibration control of landing gear components. Journal of Aerospace Engineering, 15(1), 1-9.

Lewis, F.L., \& Syrmos, V.L. (1995). Optimal control (2nd ed. ). New York, NY: John Wiley \& Sons.

Mao, W.-J., \& Chu, J. (2009). D-stability and D-stabilization of linear discrete time-delay systems with polytopic uncertainties. Automatica, 45(3), 842-846.

Moreira, M.R., Mainardi Júnior, E.I., Esteves, T.T., Teixeira, M.C.M., Cardim, R., Assunção, E., \& Faria, F.A. (2010). Stabilizability and disturbance rejection with state-derivative feedback. Mathematical Problems in Engineering, 2010, 123751.

Reithmeier, E., \& Leitmann, G. (2003). Robust vibration control of dynamical systems based on the derivative of the state. Archive of Applied Mechanics, 72(11), 856-864. 
Rossi, F.Q., \& Galvão, R.K.H. (2014). Robust predictive control of water level in an experimental pilot plant with uncertain input delay. Mathematical Problems in Engineering, 2014, 189456.

Rossi, F.Q., Galvão, R.K.H., Teixeira, M.C.M., \& Assunção, E. (2015, October 25-28). Frequency-domain robustness analysis of a discrete time state derivative feedback control law. In Proceeding of the XII Simpósio Brasileiro de Automação Inteligente ("XII Brazilian Symposium on Artificial Intelligence”) (pp. 618-623). Natal: Sociedade Brasileira de Automatica.

Rossi, F.Q., Teixeira, M.C.M., Galvão, R.K.H., \& Assunção, E. (2013, October 9-11). Discrete time design of state derivative feedback control laws. In Proceeding of the Conference on Control and Fault-Tolerant Systems (pp. 808-813). Nice: IEEE.

Silva, E.R.P., Assunção, E., Teixeira, M.C.M., \& Buzachero, L.F.S. (2012). Less conservative control design for linear systems with polytopic uncertainties via state derivative feedback. Mathematical Problems in Engineering, 2012, 315049.

Silva, E.R.P., Assunção, E., Teixeira, M.C.M., \& Cardim, R. (2013, October 9-11). Robust controller implementation via state derivative feedback in an active suspension system subject to fault. In Proceeding of the Conference on Control and Fault-Tolerant Systems (pp. 752-757). Nice: IEEE.

Silva, E.R.P., Assunção, E., Teixeira, M.C.M., Faria, F.A., \& Buzachero, L.F.S. (2011). Parameter-dependent Lyapunov functions for state derivative feedback control in polytopic linear systems. International Journal of Control, 84(8), 1377-1386.

Tseng, Y.W., \& Hsieh, J.G. (2013). Optimal control for a family of systems in novel state derivative space form with experiment in a double inverted pendulum system. Abstract and Applied Analysis, 2013, 715026.

Yang, J.N., \& Li, Z. (1991). Instantaneous optimal control with acceleration and velocity feedback. Probabilistic Engineering Mechanics, 6(3-4), 204-211.

\section{Appendices}

\section{Appendix 1 Robust model predictive control (Kothare et al., 1996)}

Consider a discrete time model of the form

$$
x((k+1) T)=\Phi x(k T)+\Gamma u(k T),
$$

where $x(k T) \in \mathbb{R}^{n}, u(k T) \in \mathbb{R}^{m}$ are the state and input vectors at time $k T$. Matrices $(\Phi, \Gamma)$ are assumed to belong to a polytope $\Omega$ with known vertices $\left(\Phi_{i}, \Gamma_{i}\right), i=1,2, \ldots$, $N$. Moreover, let $J_{\infty}(k)$ denote the following infinitehorizon cost function:

$$
\begin{aligned}
J_{\infty}(k)= & \sum_{j=0}^{\infty}\left[\|x((k+j) T \mid k T)\|_{S}^{2}\right. \\
& \left.+\| u((k+j) T) \mid k T) \|_{R}^{2}\right]
\end{aligned}
$$

where $S \in \mathbb{R}^{n \times n}$ and $R \in \mathbb{R}^{m \times m}$ are positive-definite weight matrices and $(\cdot \mid k T)$ denotes a predicted value, which is computed on the basis of the information available at time $k T$, with $x(k T \mid k T)=x(k T)$.

In view of the uncertainty in $(\Phi, \Gamma)$, the optimal control problem to be solved at each $k T$ can be formulated as

$$
\min _{u((k+j) T \mid k T), j \geq 0} \max _{(\Phi, \Gamma) \in \Omega} J_{\infty}(k)
$$

subject to input constraints of the form

$$
\left|u_{r}((k+j) T \mid k T)\right| \leq u_{r, \max }, r=1,2, \ldots, m, j \geq(\mathrm{A} 4)
$$

where $u_{r \text {, max }}$ denotes the bound on the magnitude of the $r$ th input variable.

The RMPC approach proposed by Kothare et al. (1996) consists of solving the following semidefinite programming (SDP) problem to minimise an upper bound $\gamma$ on the cost $J_{\infty}(k)$ :

$$
\min _{\gamma, Q>0, X, Y} \gamma
$$

subject to

$$
\begin{gathered}
{\left[\begin{array}{cc}
Q & x(k T) \\
* & 1
\end{array}\right] \geq 0, \quad\left[\begin{array}{cccc}
Q & 0 & 0 & \Phi_{i} Q+\Gamma_{i} Y \\
* & \gamma I & 0 & S^{1 / 2} Q \\
* & * & \gamma I & R^{1 / 2} Y \\
* & * & * & Q
\end{array}\right] \geq 0} \\
i=1,2, \ldots, N \\
{\left[\begin{array}{cc}
X & Y \\
* & Q
\end{array}\right] \geq 0, \quad X_{r r} \leq u_{r, \max }^{2}, r=1,2, \ldots, m(\mathrm{~A} 6)}
\end{gathered}
$$

where the symbol ${ }^{\star}$ is used to represent the elements below the main diagonal of a symmetric matrix and $X_{r r}$ denotes the $r$ th element in the main diagonal of $X$.

The control action at time $k T$ is then given by $u(k T)=$ $F x(k T)$, with $F=Y Q^{-1}$. At time $(k+1) T$, a new matrix $F$ is obtained by solving the SDP problem with state $x((k+$ $1) T$ ) instead of $x(k T)$ in the LMI (A6). It can be shown that the resulting closed-loop system will be robustly asymptotically stable, provided that the SDP problem is initially feasible (Kothare et al., 1996).

\section{Appendix 2 Regional pole placement (Chilali \& Gahinet, 1996)}

Consider a discrete time model of the form (A1), with polytopic uncertainty described as in Appendix 1. Moreover, let $\mathcal{D}$ be a region in the complex plane described by

$$
\mathcal{D}=\left\{z \in \mathbb{C} \mid \alpha+z \beta+\bar{z} \beta^{T}<0\right\}
$$


where $\bar{z}$ denotes the complex conjugate of $z$ and $\alpha, \beta$ are $(p \times p)$ matrices of real-valued coefficients, with $\alpha$ symmetrical.

As shown in Chilali and Gahinet, 1996, if there exist matrices $X=X^{T} \in \mathbb{R}^{n \times n}$ and $L \in \mathbb{R}^{m \times n}$ such that the following LMIs are satisfied:

$\alpha \otimes X+\beta \otimes\left(\Phi_{i} X+\Gamma_{i} L\right)+\beta^{T} \otimes\left(\Phi_{i} X+\Gamma_{i} L\right)^{T}<0$, $i=1, \ldots, N$

$X>0$

then a control law of the form $u(k T)=F x(k T)$, with $F=$ $L X^{-1}$, will place the closed-loop poles inside $\mathcal{D}$, for any
$(\Phi, \Gamma) \in \Omega$. (The symbol $\otimes$ denotes the Kronecker product of matrices.)

A particular case consists of placing the closed-loop poles inside a circle of radius $r$ and centre $\left(\chi_{0}, 0\right)$, i.e. $\mathcal{D}=$ $\left\{z=(\chi+j v) \mid\left(\chi-\chi_{0}\right)^{2}+v^{2}<r^{2}\right\}$. For this purpose, Schur's complement can be used to rewrite the inequality $\left(\chi-\chi_{0}\right)^{2}+v^{2}<r^{2}$ as

$$
\left[\begin{array}{cc}
-r & -\chi_{0}+z \\
-\chi_{0}+\bar{z} & -r
\end{array}\right]<0
$$

which can be cast into the form $\alpha+z \beta+\bar{z} \beta^{T}<0$ of (B1), with $\alpha$ and $\beta$ given by

$$
\alpha=\left[\begin{array}{cc}
-r & -\chi_{0} \\
-\chi_{0} & -r
\end{array}\right], \quad \beta=\left[\begin{array}{ll}
0 & 1 \\
0 & 0
\end{array}\right]
$$

